\title{
The environment of active galaxies in the SDSS-DR4
}

\author{
G. Sorrentino, M. Radovich, and A. Rifatto
}

\author{
INAF - Osservatorio Astronomico di Capodimonte, via Moiariello, 16, 80131 Napoli, Italy \\ e-mail: [gsorrent;radovich;rifatto]@na.astro.it
}

Received 14 October 2005 / Accepted 14 January 2006

ABSTRACT

\begin{abstract}
Aims. We study the environment of active galaxies and compare it with that of star-forming and normal galaxies.
Methods. From the Fourth Data Release (DR4) of the Sloan Digital Sky Survey (SDSS) we extracted the galaxies in the redshift range $0.05 \leq z \leq 0.095$ and with $M(r) \leq-20.0$ (that is, $M^{*}+1.45$ ). Emission-line ratios and/or widths were used to separate active galactic nuclei (AGNs) from star-forming galaxies (SFGs); AGNs were classified as Seyfert-1 (Sy1) and Seyfert-2 (Sy2) galaxies according to emission-line widths. The environmental properties, as defined by a density parameter and the number of companions, are compared for the different types of galaxies, taking the morphological type of the host galaxies into account.

Results. We find no difference in the large-scale environment of Sy1 and Sy2 galaxies; however, a larger fraction of Sy2 ( 2\%) than Sy1 $(\sim 1 \%)$ is found in systems that are smaller than $r_{\max } \leq 100 \mathrm{kpc}$, mainly in low-density environments (pairs or triplets). For comparison, this fraction is $\sim 2 \%$ for star-forming galaxies and $\sim 1 \%$ for normal galaxies.

Conclusions. We find no evidence of a relation between large-scale environment properties and activity. If activity and environment are related, this more likely occurs on small scales (e.g. galaxy interaction, merging).
\end{abstract}

Key words. galaxies: active - galaxies: Seyfert - galaxies: starburst

\section{Introduction}

The availability of surveys that provide very large databases (e.g., Las Campanas Redshift Survey: Shectman et al. 1996; 2dF Galaxy Redshift Survey: Colless et al. 2001; Sloan Digital Sky Survey: York et al. 2000) allows for robust statistic analyses of galaxy properties, such as their clustering, luminosity, starformation rate, and environment. As a consequence, the data from these surveys are leading to significant advances in the study of galaxy formation and evolution (Kauffmann et al. 1999; Benson et al. 2002).

One major topic that can be addressed is the relationship between galaxy environment and activity (SFGs and AGNs). For example, the density in the environment of SFGs is more typical of field galaxies than cluster galaxies, which suggests that star-formation is related more to local processes such as tidal triggering. Moreover, the role of interactions in triggering nuclear starbursts is now widely accepted (e.g. Storchi-Bergmann et al. 2001), and an increment of the star-formation rate is observed for galaxies in close-pair systems (Lambas et al. 2003; Sorrentino et al. 2003; Nikolic et al. 2004).

The situation is less clear for AGNs. Stauffer (1982) was one of the first to point out that Seyfert galaxies usually occur in groups, and Dahari $(1984,1985)$ suggested that these galaxies have an excess of companions compared to normal galaxies. This result has been confirmed by several studies (e.g., Laurikainen et al. 1994; Rafanelli et al. 1995) but also contradicted by others (e.g., Fuentes-Williams \& Stocke 1988; de Robertis et al. 1998) in which no detectable excess of companions around Seyfert galaxies is found. Schmitt (2001) found that there is no difference in the fraction of galaxies with companions among different activity types if we consider only galaxies with similar morphological types. This result is consistent with those found by Fuentes-Williams \& Stocke (1988) and de Robertis et al. (1998) and also with more recent results on clustering of low-luminosity AGNs at higher redshifts (Brown et al. 2001; Schreier et al. 2001). Other studies of Seyfert galaxies indicate that Sy2 have a larger number of companions when compared with normal galaxies, while Sy1 do not (Laurikainen \& Salo 1995; Dultzin-Hacyan et al. 1999; Koulouridis et al. 2005). As for environment properties, according to de Robertis et al. (1998), within $50 \mathrm{kpc}$ Sy2 inhabit richer environments than do Sy1. On larger scales (<1 Mpc) Koulouridis et al. (2005) found that Sy2 reside in less dense large-scale environments than Sy1, but this is probably related to the different morphological types of the host galaxies.

According to the so-called unified model (Antonucci 1993), different properties observed in AGNs are not due to intrinsic differences: in particular, an AGNs may appear as a type 1 or type 2 depending on the orientation to our line of sight of a circumnuclear torus of dust and gas. Indeed, the unified model does not imply that other processes may not occur in the nuclear region, which may even prevail for nearby, low-luminosity AGNs (Seyfert galaxies) or for dust-obscured AGNs. Schmitt (2001) suggests that interactions are important for triggering activity but that a starburst (SB) may prevail in the earlier phase, hiding any AGNs that might be present. Storchi-Bergmann et al. (2001) propose an evolutionary link from SFGs to Sy2 galaxies, driven by interaction. They find a correlation between the presence of companions, the inner morphology, and the incidence of recent star-formation, suggesting an evolutionary scenario in which the interaction is responsible for sending gas inward, which both feeds the AGNs and triggers star-formation. The SB then fades with time and the composite Sy $2+$ SB nucleus evolves into a "pure" Seyfert nuclei that may be of type 1 or 2 in agreement with the unified model. The existence of two different Sy2 population was finally suggested by Tran (2003) from the absence of detectable polarized broad lines in a fraction of Sy2 and a 
comparison of their properties with those of Sy1 and Sy2 with polarized broad lines.

A crucial question to be addressed is therefore whether AGNs and SFGs are found in similar environments, and in particular if there are differences in the environments of type- 1 and type-2 AGNs.

In this paper we shall use the Fourth Data Release (DR4) of the Sloan Digital Sky Survey (SDSS) to investigate the environment of a complete sample of active galaxies. Spectroscopic data will be used to classify them as SFGs, type-1, or type-2 AGNs, and to compare their environmental properties. In addition, photometric parameters will be used for a morphological classification (early and late-type) of the AGNs host galaxies.

The paper is organized as follows. In Sects. 2 and 3, we describe the data set and the extraction of the samples. The algorithms used to find the number of neighbours and compute the density are outlined in Sect. 4. The results are presented and discussed in Sect. 5, while the conclusions are in Sect. 6.

\section{SDSS-DR4 spectroscopic survey}

The Sloan Digital Sky Survey (SDSS, York et al. 2000; Abazajian et al. 2004) is a photometric and spectroscopic survey that will map about one quarter of the entire sky outside the Galactic plane and will collect spectra of about $10^{6}$ galaxies, $10^{5}$ quasars, 30000 stars and 30000 serendipity targets.

Photometry is available in $u, g, r, i$, and $z$ bands (Fukugita et al. 1996; Gunn et al. 1998), while the spectroscopic data are obtained with a pair of multi-fiber spectrographs. In the fourth data release (DR4, http://www.sdss.org/dr4), the spectroscopic survey covers an area of 4681 square degrees. The spectra cover the spectral range $3800<\lambda<9200 \AA$, with a resolution of $1800<\lambda / \Delta \lambda<2100$, and give an rms redshift accuracy of $30 \mathrm{~km} \mathrm{~s}^{-1}$, to an apparent magnitude limit (Petrosian magnitude) of $r=17.77$. The fiber diameter is $0.2 \mathrm{~mm}$ ( $3^{\prime \prime}$ on the sky), and adjacent fibers cannot be located more closely than $55^{\prime \prime}$ on the sky $\left(\sim 110 \mathrm{kpc}\right.$ at $z=0.1$ with $H_{0}=$ $75 \mathrm{~km} \mathrm{~s}^{-1} \mathrm{Mpc}^{-1}$ ) during the same observation. Multiple targets closer than this distance are said to "collide". Starting from the spectroscopic SDSS-DR2, a tiling method has been developed in order to optimize the placement of fibers on individual plates, as well as the placement of plates relative to each other. This method allows a sampling rate of more than $92 \%$ for all targets, and more than $99 \%$ for the set of targets that do not collide, with an efficiency greater than $90 \%$ (Blanton et al. 2003b; www.sdss.org/dr4/algorithms/tiling.html). The spectroscopic SDSS-DR4 catalog contains 849920 spectra, among which 565715 are classified as galaxies and 76483 are classified as quasars.

Data have been obtained from the SDSS database (http://www.sdss.org/dr4) using the CasJobs facility (http://casjobs.sdss.org/casjobs/).

\section{Sample definition}

The definition of a volume-limited sample was done as in Miller et al. (2003). We considered all galaxies brighter than $M(r)=$ -20.00 , that is, $M^{*}(r)+1.45$ with $M^{*}(r)=-20.8+5 \log h$ (Blanton et al. 2001, 2003a). This translates into a redshift range $0.05<z<0.095$ (Fig. 1, left panel). The lower redshift limit is aimed at minimizing the aperture bias (Gómez et al. 2003) due to large nearby galaxies. The upper limit corresponds to where the luminosity limit equals the apparent magnitude limit ( $r=17.77 \mathrm{mag}$ ) of the SDSS (Strauss et al. 2002). In this way we selected 90886 galaxies. Concerning those targets closer than 55", we verified that a significant fraction is indeed included in the spectroscopic catalog. To this aim, we first calculated the number of neighbours detected within 55" around each galaxy brighter than $r=17.77 \mathrm{mag}$, using the full DR4 photometric catalog. The same number was then computed taking only galaxies with a spectroscopic redshift. In all cases we obtained $\sim 91 \%$ of the galaxies are detected both in the photometric and in the spectroscopic catalogs, in agreement with Blanton et al. (2003b).

Galaxies with no detectable emission-lines, which are expected to have a morphological type earlier than $\mathrm{Sa}$, are defined as passive galaxies (PGs). There are 16403 PGs out of 90886 galaxies $(\sim 18 \%)$. Emission-line galaxies are defined as galaxies with one or more emission-lines having $I_{\lambda} / \sigma_{I_{\lambda}}>$ 2 , where $I_{\lambda}$ is the emission-line flux and $\sigma_{I_{\lambda}}$ its uncertainty. This gives 57952 galaxies $(\sim 64 \%)$. The remaining $18 \%$ are composed of galaxies with a large error in the detected lines. These galaxies are not taken into account because the large error $\left(I_{\lambda} / \sigma_{I_{\lambda}}<2\right)$ does not allow a certain classification.

AGNss and SFGs were first separated using the theoretical line-ratio models proposed by Kewley et al. (2001):

$$
\begin{aligned}
\log \left(\frac{[\mathrm{OIII}] \lambda 5007}{\mathrm{H}_{\beta}}\right) & =\frac{0.61}{\log \left([\mathrm{NII}] \lambda 6583 / \mathrm{H}_{\alpha}\right)-0.47}+1.19 \\
\log \left(\frac{[\mathrm{OIII}] \lambda 5007}{\mathrm{H}_{\beta}}\right) & =\frac{0.72}{\log \left(\frac{[\mathrm{III}](\lambda \lambda 6717,6731)}{\mathrm{H}_{\alpha}}\right)-0.32}+1.30 \\
\log \left(\frac{[\mathrm{OIII}] \lambda 5007}{\mathrm{H}_{\beta}}\right) & =\frac{0.73}{\log \left([\mathrm{OI}] \lambda 6300 / \mathrm{H}_{\alpha}\right)+0.59}+1.33
\end{aligned}
$$

These ratios were chosen to give the best separation of the two classes of objects; the $[\mathrm{OIII}] / \mathrm{H}_{\beta}$ ratio is an indicator of the mean level of ionization and temperature, while the $[\mathrm{NII}] / \mathrm{H}_{\alpha},[\mathrm{OI}] / \mathrm{H}_{\alpha}$ and $[\mathrm{SII}] / \mathrm{H}_{\alpha}$ ratios are indicators of the relative importance of the partially ionized region produced by high-energy photoionization. All ratios are based on lines close in wavelength so the correction for dust reddening is negligible.

We removed those sources whose line ratios fall close to the border line to avoid possible "ambiguous" cases. This was done by keeping only those galaxies for which part of the $\sigma$ error bar associated to the logarithm of the detected $[\mathrm{OIII}] / \mathrm{H}_{\beta}$ and $[\mathrm{NII}] \lambda 6583 / \mathrm{H}_{\alpha}$, or $[\mathrm{SII}](\lambda \lambda 6717,6731) / \mathrm{H}_{\alpha}$, or $[\mathrm{OI}] \lambda 6300 / \mathrm{H}_{\alpha}$, respectively, lie within the theoretical uncertainty of the model $\left(\sigma_{\text {mod }}=0.1 \mathrm{dex}\right)$ in both $x$ and $y$ directions (Fig. 2). So we take into account only the galaxies whose line ratios, considering their error bars as well, lie outside the uncertainty region. We used all the diagnostic ratios when available, with the minimum requirement of the presence of $\mathrm{H}_{\alpha}, \mathrm{H}_{\beta}$, [OIII] $\lambda 5007$, and $[\mathrm{NII}] \lambda 6583$.

AGNss were classified as Sy1 if $F W H M\left(\mathrm{H}_{\alpha}\right)>$ 1.5 FWHM([OIII] 25007$)$, or as Sy2 otherwise. We also classified as Sy1 all the emission-line galaxies having at least $\mathrm{H}_{\alpha}$ and [OIII] $\lambda 5007$ emission-lines with $F W H M\left(\mathrm{H}_{\alpha}\right)>1200 \mathrm{~km} \mathrm{~s}^{-1}$ and $F W H M([\mathrm{OIII}] \lambda 5007)<800 \mathrm{~km} \mathrm{~s}^{-1}$, independent of line ratios: these limits were empirically found by looking at the distribution of the FWHMs (Fig. 3) and examining the spectra. The final sample of AGNs consists of 1829 galaxies $(\sim 2 \%)$, 725 Sy1 and 1104 Sy2; the number of SFGs is $6061(\sim 7 \%)$.

Unclassified Emission-Line Galaxies (UELGs) are those galaxies that are not univocally classified either as AGNs or SFGs according to all the measured line ratios: there are 50062 UELGs $(\sim 55 \%)$. As a consequence of the morphologydensity relation (Dressler 1980), the morphological type of the 

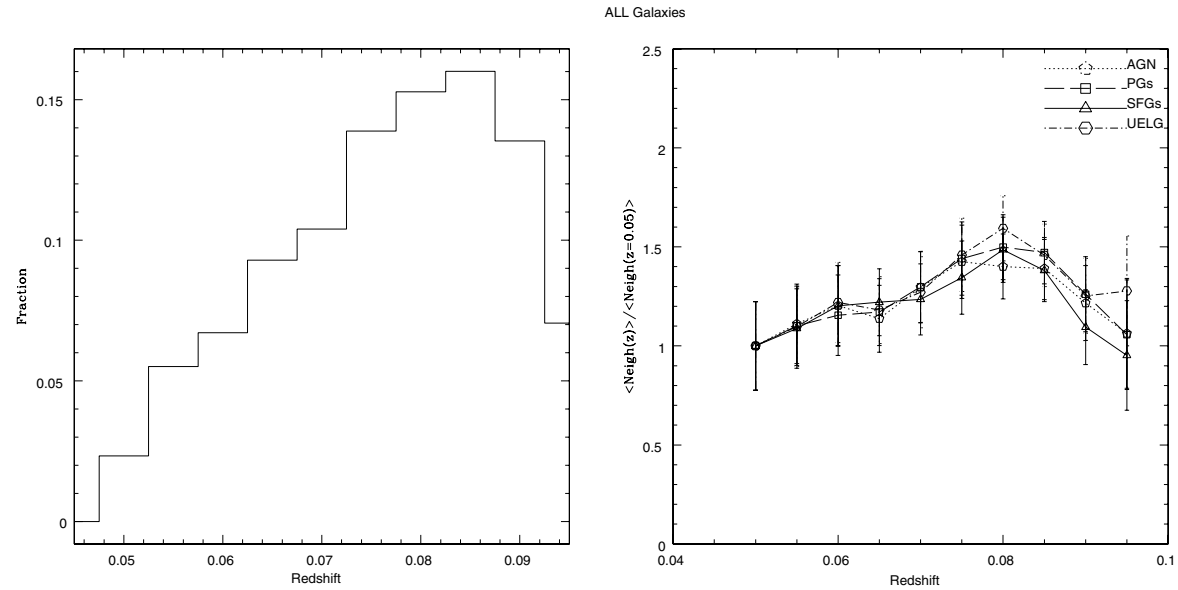

Fig. 1. Redshift distribution (left) and mean number of neighbours vs. redshift (right).

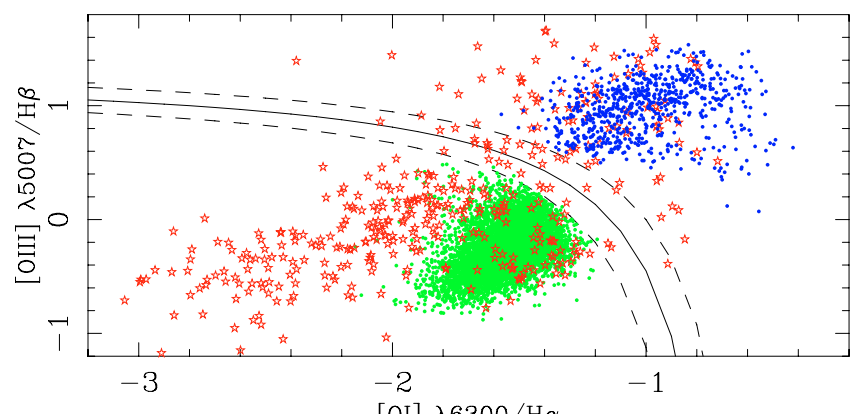

[OI] $\lambda 6300 / \mathrm{H} \alpha$

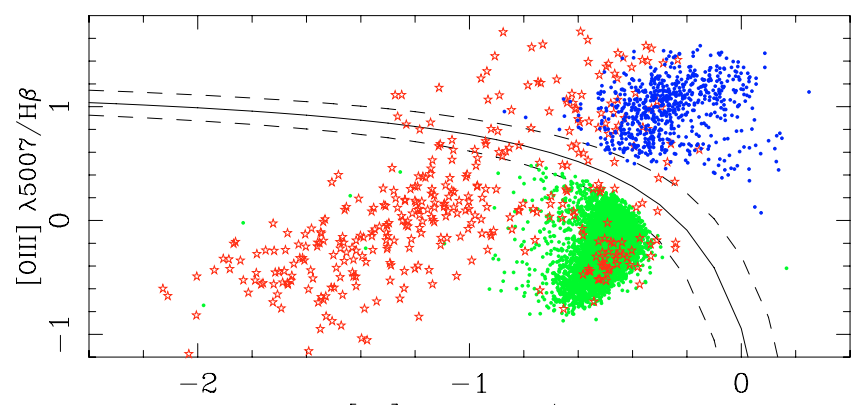

[SII] $\lambda \lambda 6716,31 / \mathrm{H} \alpha$

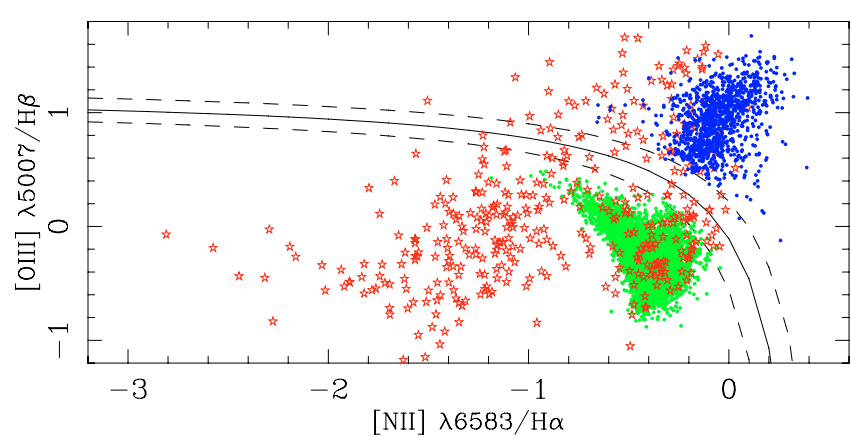

Fig. 2. Diagnostic diagrams for Sy1 (stars), Sy2, and star-forming galaxies.

host galaxy must be considered for a proper comparison of the environmental properties of AGNs, SFGs, and PGs. Some authors have proposed that the presence of the active nucleus may alter the morphological properties of the host galaxy (e.g.

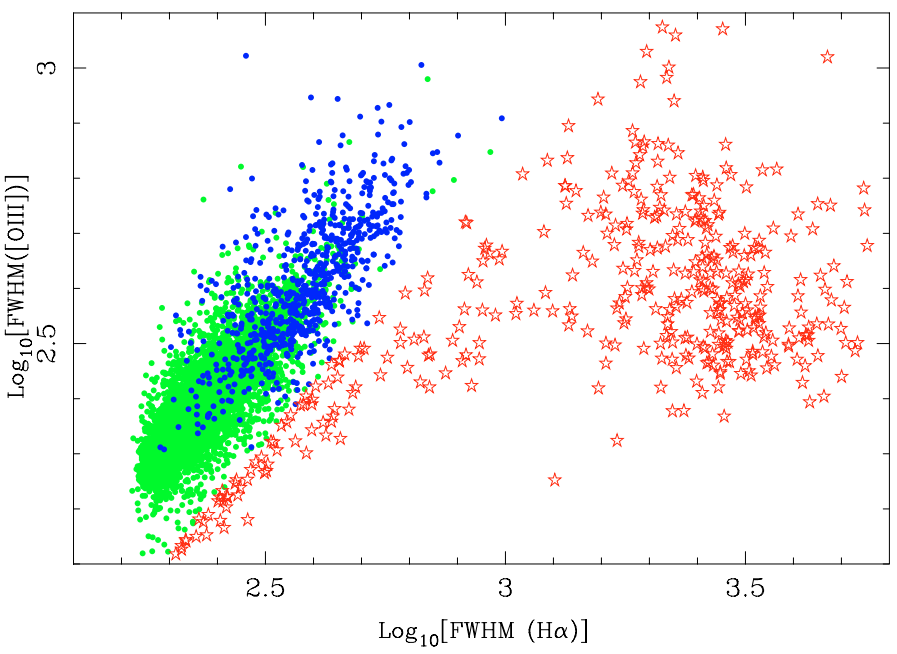

Fig. 3. $F W H M([\mathrm{OIII}])$ and $F W H M\left(\mathrm{H}_{\alpha}\right)$ in emission-line galaxies, showing the clear separation of broad (Sy1) and narrow-line (Sy2 and SFGs) galaxies with the criteria adopted in the paper. Symbols are as in Fig. 2.

Walker et al. 1996); however, according to Martini et al. (2003), there is no systematic difference in the circumnuclear environments of active and inactive galaxies (e.g., an excess of nuclear bars and/or nuclear dust spirals). For this reason, we separated both active and non active galaxies according to their morphological type, defined by the two parameters ECLASS and FRACDEV provided by the SDSS. FRACDEV is a photometric parameter providing the weight of a de Vaucouleurs component in the best composite exponential + de Vaucouleurs models, and ECLASS is a spectroscopic parameter giving the spectral type from a principal component analysis. Early-type galaxies $(\mathrm{E}+$ S0) were selected following the criteria adopted by Bernardi et al. (2005): $\operatorname{FRACDEV}(r)>0.8$ and ECLASS $<0$. Late-type galaxies (Sa and later) were selected when either ECLASS $\geq 0$ or $\operatorname{FRACDEV}(r)<0.5$. In this way we excluded from our analysis all the galaxies with $\operatorname{FRACDEV}(r) \geq 0.5$ and ECLASS $<0$, for which an unambiguous classification is not possible. These selection criteria were used to separate early- and late-type galaxies for Sy2, PGs, SFGs, and UELGs. In the case of Sy1, it is not possible to use the spectral type, because the continuum is modified by the non-termal component, and we therefore rely on the FRACDEV parameter only: Sy1 are classified as "early" if $\operatorname{FRACDEV}(r)>0.8$, and as "late" if $\operatorname{FRACDEV}(r)<0.5$. For the 
Table 1. Median and rms of $r$-band absolute magnitudes for AGNs and normal galaxies.

\begin{tabular}{cccc}
\hline \hline Type & $N$ & $\langle M(r)\rangle$ & $\sigma$ \\
\hline Sy1 early & 553 & -21.2 & 0.6 \\
Sy1 late & 71 & -21.0 & 0.6 \\
Sy2 early & 297 & -21.0 & 0.5 \\
Sy2 late & 628 & -20.8 & 0.5 \\
PGs (early) & 16403 & -20.7 & 0.6 \\
UELG (late) & 20141 & -20.6 & 0.5 \\
SFGs (late) & 5920 & -20.5 & 0.4 \\
\hline
\end{tabular}

selection of the control samples, we first verified that the redshift distribution of neighbour galaxies is the same for AGNs, SFGs, UELGs, and PG, as can be seen from Fig. 1, right panel. As for AGNs, the luminosity is biased by the contribution from the nucleus (see Table 1), and control samples were not matched in absolute magnitudes. Instead, we proceeded as Krongold et al. (2002) and Koulouridis et al. (2005), who matched the control samples by the diameter size distribution. We randomly extracted early-type (PGs) and late-type (SFGs and UELGs) galaxies to build control samples with the same distribution in diameter $\left(D_{25}\right)$ of early/late-type AGNs.

\section{Research algorithm and density parameter}

As the aim of the paper is to analyze the environment of active galaxies in both poor and rich systems, we can now look at some of the many possible approaches to carry it out.

One of the methods used the most is based on determining the density evaluated from the distance to the $N$ th companion. Most authors use the 10th nearest neighbour (Dressler 1980; Miller et al. 2003; Gómez et al. 2003; Carter et al. 2001; Balogh et al. 2004); as a consequence this method is suitable for environments of systems with many galaxies $(N>10)$, e.g. rich groups or clusters (Dressler 1980), but it does not take into account the small systems with $N_{\text {neigh }}<10$ (pairs and poor groups).

In this paper the density parameter is defined as:

$\Sigma=\frac{N_{\text {neigh }}}{\pi r_{\max }^{2}}$

where $N_{\text {neigh }}$ is the number of neighbouring galaxies, and $r_{\max }$ is the distance between the galaxy and the most distant companion. A galaxy $j$ is considered as a neighbour of a galaxy $i$ if:

- $D_{i j} \leq D_{\max }$

- $c\left|z_{i}-z_{j}\right| \leq 1000 \mathrm{~km} \mathrm{~s}^{-1}$

where $D_{i j}$ is the projected distance between the two galaxies, and $\left|z_{i}-z_{j}\right|$ is their redshift difference. Then $D_{i j}$ is computed from the angular separation $\theta_{i j}$ and the redshift $z_{i}$, assuming $H_{0}=75 \mathrm{~km} \mathrm{~s}^{-1} \mathrm{Mpc}^{-1}$. Figure 4 displays the distribution of the redshift differences. It shows that a negligible fraction of galaxies is found for $c\left|z_{i}-z_{j}\right|>1000 \mathrm{~km} \mathrm{~s}^{-1}$, which is the limit usually adopted for selecting cluster or galaxy group members in the velocity space (Fadda et al. 1996; Wilman et al. 2005).

The upper distance limit is the typical size of a cluster, being $D_{\max }=1 h^{-1} \mathrm{Mpc} \sim r_{\text {Abell }}$ (Abell 1958). The distribution in the number of neighbour galaxies and the average density parameter for all galaxies are displayed in Fig. 5. From the right panel of this figure, it is evident that there is a linear correlation between $N_{\text {neigh }}$ and $\langle\Sigma\rangle$ for systems with $N_{\text {neigh }}>3$. This implies that for these systems the density parameter does not depend

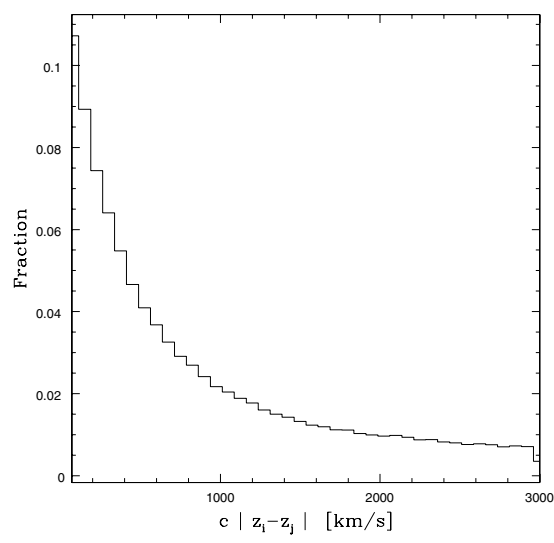

Fig. 4. Distribution of the velocity difference of neighbouring galaxies.

on $r_{\text {max }}$. Therefore, for systems with $N_{\text {neigh }}>3$, our definition of the density parameter is equivalent to taking a fixed surface area; for small systems (galaxy pairs and triplets), the density is linked to the physical size.

The properties of a small-scale environment ( $r \leq 100 \mathrm{kpc})$ were investigated considering two different cases: a) systems with $r_{\max } \leq 100 \mathrm{kpc}$, hereafter defined as close systems; and b) systems with at least one companion within $100 \mathrm{kpc}$. In the following discussion, the median of the surface density is computed rather than the average, to minimize the effect of few systems with very high surface density.

\section{Results and discussion}

In our sample, the overall fraction of galaxies with a definite AGNs is $\sim 2 \%$. This percentage is significantly different from the fraction of AGNs found by Miller et al. (2003) (20-40\%) and by Carter et al.(2001) ( 17\%). It is comparable to the values found by Dressler et al. (1985) (5\% in the field sample and $1 \%$ in the cluster sample), by Huchra \& Burg (1992) (1.3\%), by Ivezić et al. (2002) using the SDSS data (5\%), and by Maia et al. (2004) (3-4\%). It, however, should be taken into account that our AGNs classification was done using all diagnostic ratios when available, while other authors use only the first of these $\left(\frac{[\mathrm{OIII}] \lambda 5007}{\mathrm{H}_{\beta}}\right.$ vs. $\left.\frac{[\mathrm{NII}] \lambda 6583}{\mathrm{H}}\right)$.

The AGNs fraction we find is therefore an underestimate of the true value, as we lose an unknown fraction of AGNs (faint lines or ambiguous diagnostic ratios, see previous section). Figure 6 displays the fraction of galaxies as a function of $\Sigma$. Two different trends are found. For values of $\Sigma<10$ (Fig. 6, left panel), the fraction of SFGs decreases with density, whereas the fraction of PGs increases. The same trends were found by Miller et al. (2003), in agreement with the SFR-density relation (Gomez et al. 2003) and the morphology-density relation (Dressler 1980). The opposite result is found for $\Sigma>10$ : in dense environments the fraction of SFGs increases and the fraction of PGs decreases. Data from the 2dFGRS (Lambas et al. 2003; Sorrentino et al. 2003) and the SDSS-DR1 (Nikolic et al. 2004) indicate that star-formation is enhanced in galaxy pairs and in particular that it increases for close pairs. This is consistent with what we see, since enhanced star-formation implies a higher probability that a galaxy is classified as an SFG from its line ratios. The fraction of AGNs does not change with density, in agreement with the result found by Carter et al. (2001) and Miller et al. (2003). The main environmental parameters (number of galaxies, percentage, and surface density) are displayed 

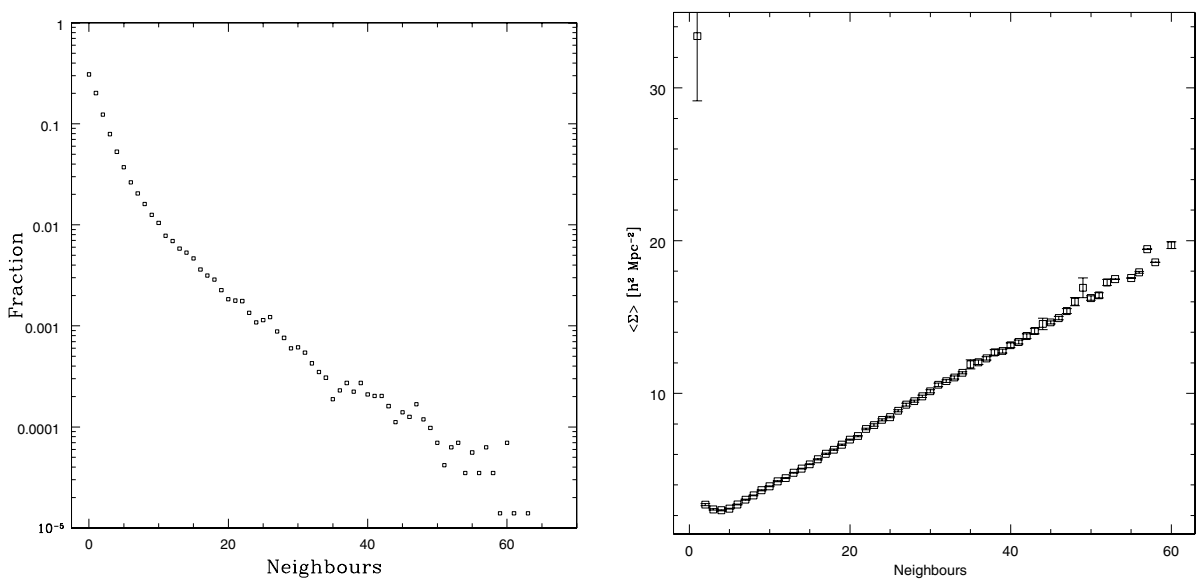

Fig. 5. The distribution of the number of neighbours (left), and the relation between number of neighbours and the average density parameter (right) for all the galaxies of our sample.
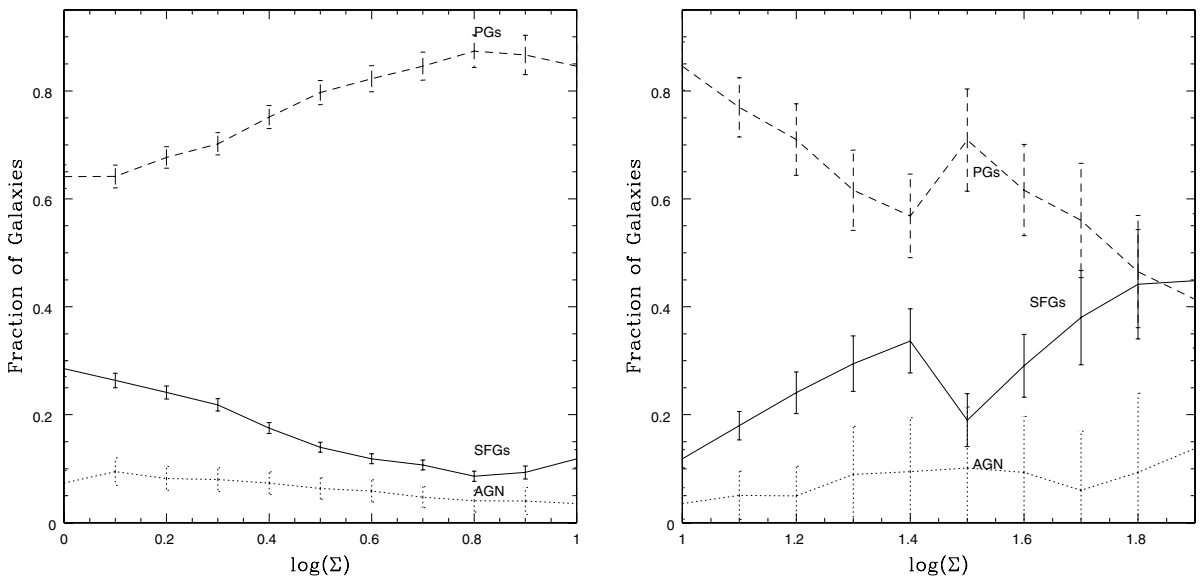

Fig. 6. Fraction of galaxies vs. density in two ranges of density. The left panel is comparable with other previous works on rich systems

in Figs. 7-10, and in Table 2. We consider separately close systems $\left(r_{\max } \leq 100 \mathrm{kpc}\right)$, since for these systems the analysis may be partly biased by the limit on the fiber separation (see Sect. 2).

We first examined the environmental properties for the full AGNs sample. No difference in the environment of Sy1 and Sy2 is evident: the median surface density is $\langle\Sigma\rangle \sim 1.5$ (Fig. 7) as in SFGs, whereas it is higher in PGs $(\langle\Sigma\rangle \sim 2.5)$. In addition, PGs can be found in richer systems $\left(N_{\text {neigh }} \leq 60\right)$ than both SFGs $\left(N_{\text {neigh }} \leq 35\right)$ and AGNs $\left(N_{\text {neigh }} \leq 30\right)$ (Fig. 8). As it concerns close systems, which are mainly pairs, we find a higher fraction of Sy2 $(\sim 2 \%)$ compared to Sy1 $(\sim 1 \%)$. This result is in agreement with Dultzin-Hacyan et al. (1999).

We then examined (Table 3 ) the fraction of systems with at least one close neighbour $(r<100 \mathrm{kpc})$. We performed a Kolmogorov-Smirnov (K-S) test to check whether the frequency distributions in AGNs and control samples are the same. If we consider all systems independently from the number of neighbours, we find a low $(<4 \%)$ probability that the frequency distribution in Sy1 and Sy2 is the same. This increases to $\sim 30 \%$ and $\sim 99 \%$ if we exclude pairs and both pairs and triplets, respectively. The comparison with control samples shows that the frequency in Sy2 is statistically consistent $(>20 \%)$ with that in SFGs. For Sy1, the distribution is not consistent with either SFGs or PGs; it is consistent ( 90\%) with SFGs if we exclude pairs and triplets.

The same analysis was then carried out taking the morphological type of the host galaxies into account. The properties of

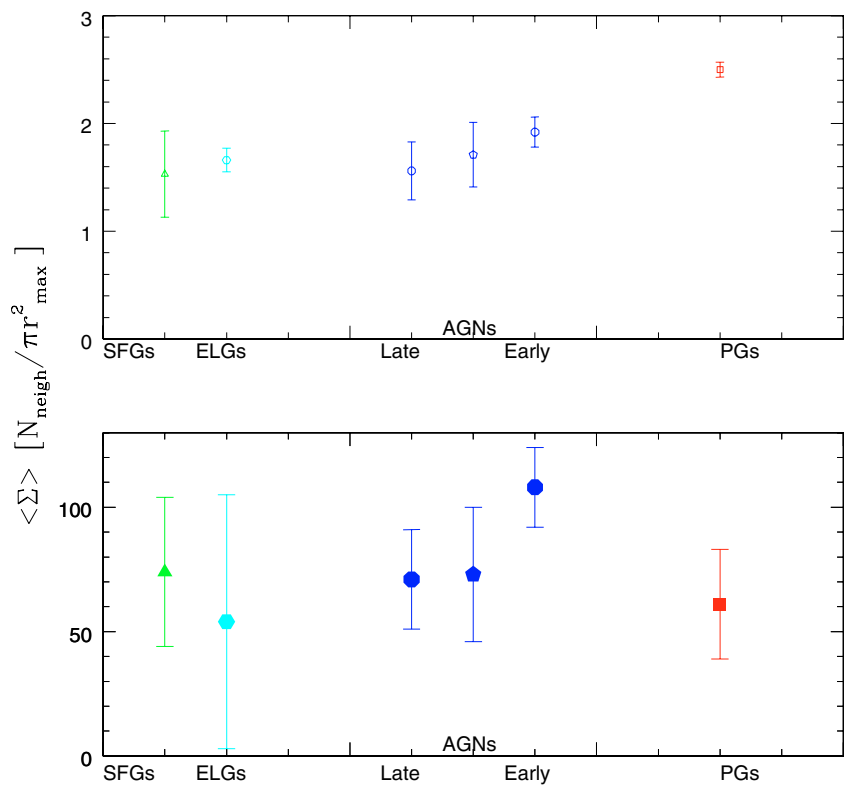

Fig. 7. Mean surface density parameter for PGs, SFGs, and AGNs. Top: all systems with $r_{\max }>100 \mathrm{kpc}$. Bottom: close systems $\left(r_{\max }<\right.$ $100 \mathrm{kpc})$.

early- and late-type Sy1 and Sy2 were compared with those of the control samples defined above (PGs, UELGs, and SFGs). 

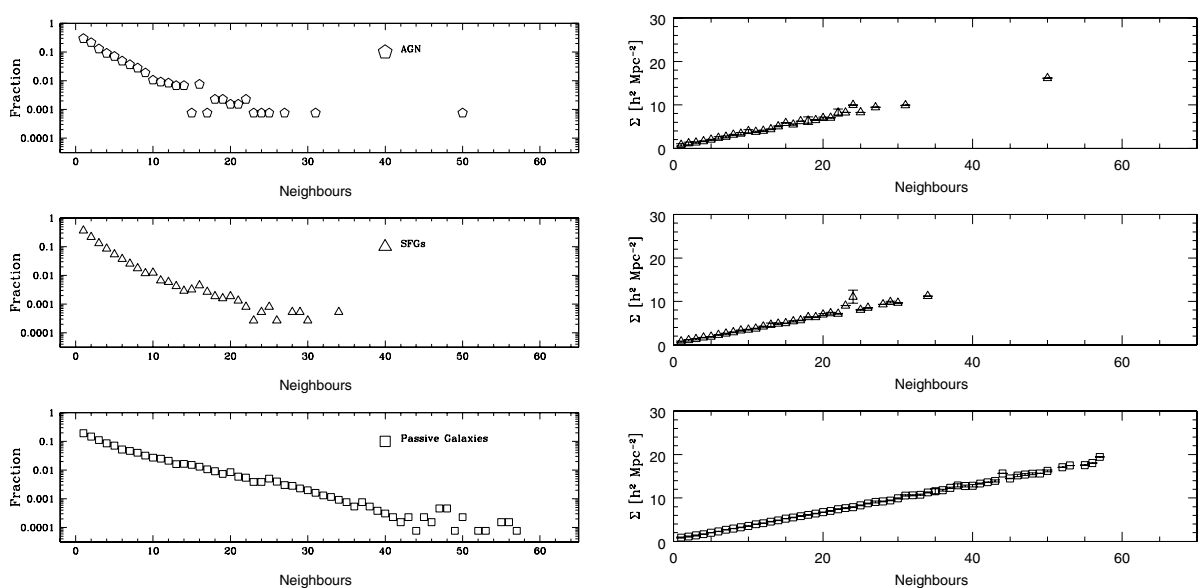

Fig. 8. Comparison of environmental properties for PGs, SFGs, and AGNs.
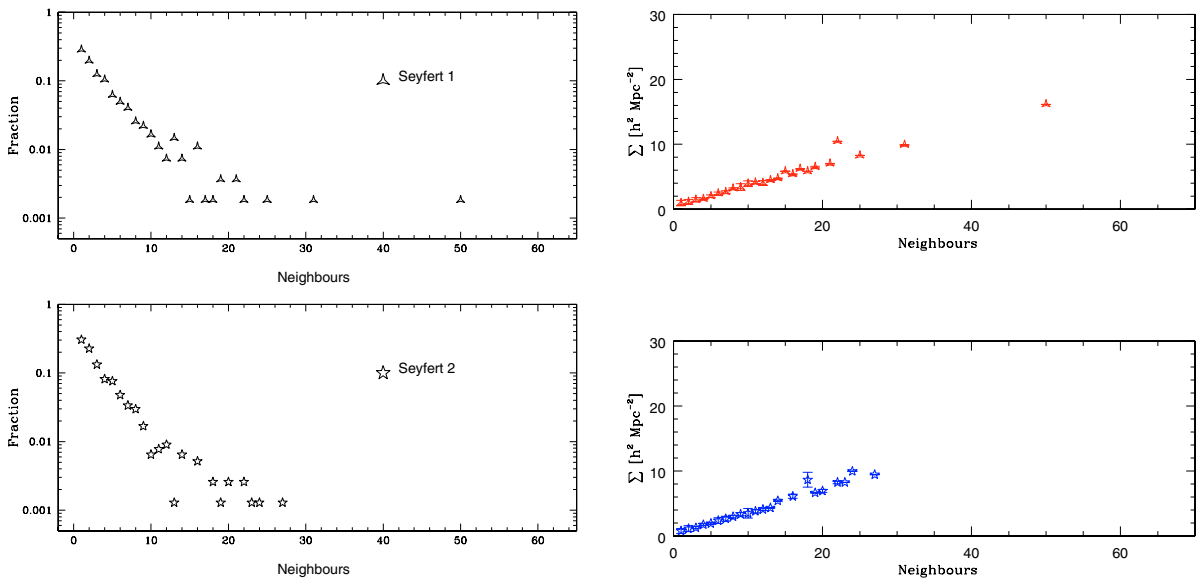

Fig. 9. Environmental properties for $\mathrm{Sy} 1$ and $\mathrm{Sy} 2$ galaxies.

The comparison of Sy1 and Sy2 galaxies with the same morphological type (Table 2 and Fig. 10) indicates that the distribution in the number of neighbour galaxies is very similar, as confirmed by the K-S test. The median surface density in early-type AGNs $(\langle\Sigma\rangle \sim 2)$ is slightly higher than in late-type AGNs $(\langle\Sigma\rangle \sim 1.5)$, as expected. The distribution in number of neighbours of early and late Seyfert is similar to that of PGs and UELGs/SFGs, respectively. The values of the median surface density in all bins are also comparable. We therefore conclude that there is no strong evidence of a denser environment in AGNs compared to normal galaxies, in agreement with the results of Schmitt (2001). The morphological separation does not, however, change the difference in close systems between Sy1 and Sy 2 galaxies. In fact, the fraction of close systems found in early/late-type Sy1 is the same as for PGs and UELGs $(\sim 1 \%)$; the fraction found for early/late-type Sy2 is in agreement with what is found in SFGs $(\sim 2 \%)$.

As it concerns the frequency of systems with close neighbours, we find the same distribution (>90\%) if we compare Sy 1 and Sy2 in early-type galaxies. The distribution for Sy1 and Sy2 in late-type galaxies is consistent if we exclude pairs $(>20 \%)$, or pairs and triplets $(>90 \%)$. The comparison with the control samples confirms what is found above: the distribution of Sy2 in both late- and early-type galaxies is consistent with SFGs, which is true for Syl as well, if pairs are not included.

We finally checked whether there is an excess of isolated or paired systems for Sy 2 compared to Sy 1 galaxies, independent of their size. To this aim we computed the fraction $f_{\text {iso }}\left(f_{\text {pair }}\right)$

of isolated (paired) Sy1 and Sy2 galaxies to the total number of isolated (paired) Seyfert galaxies. The same values as for the total population are found: $f_{\text {iso }}(\mathrm{Sy} 1) \simeq f_{\text {pair }}(\mathrm{Sy} 1) \simeq f(\mathrm{Sy} 1) \sim$ $0.4, f_{\text {iso }}(\mathrm{Sy} 2) \simeq f_{\text {pair }}(\mathrm{Sy} 2) \simeq f(\mathrm{Sy} 2) \sim 0.6$. If we consider only close systems, we find $f_{\mathrm{cs}}(\mathrm{Sy} 1)=0.3$ and $f_{\mathrm{cs}}(\mathrm{Sy} 2)=0.7$.

We conclude that there is no difference in the large-scale environments of Sy 1 and Sy2 and there is no contradiction with the Unified Model.

A similar result was found by Koulouridis et al. (2005); that is, any difference in the large-scale environment of Sy1 and Sy2 is related to the morphological type of the host galaxy rather than to the activity. The same authors conclude that $\mathrm{Sy} 2$ galaxies have close companions more frequently than Sy1 galaxies, in agreement with de Robertis (1998). We obtain the same result, but only for galaxies in low-density environments (pairs and triplets). We did not find any difference in richer systems.

\section{Conclusions}

In this paper we have studied the environment of active galaxies (AGNs and SFGs) in the SDSS-DR4 in the redshift range $0.05 \leq$ $z \leq 0.095$ and with $M(r) \leq-20.0$. The presence of emissionlines was used to separate active galaxies from PGs. The AGNs and SFGs were then separated according to their emission line ratios. AGNs were further separated into Sy1 and Sy2 galaxies using the width of the emission-line [OIII] $\lambda 5007$ with respect to the Balmer lines $\left(\mathrm{H}_{\alpha}, \mathrm{H}_{\beta}\right)$. 
Table 2. Environmental properties for AGNs and control samples of normal galaxies with the same distribution in the galaxy diameter. The number $(N)$, the fraction $(f)$ of galaxies, and the median surface density $(\Sigma)$ with the associated errors $(\sigma)$ are given for different bins in the number of neighbour galaxies.

\begin{tabular}{|c|c|c|c|c|c|c|c|c|c|c|c|c|c|c|c|}
\hline \multirow{2}{*}{ Neighbours } & \multicolumn{5}{|c|}{$\overline{\text { Sy1 }}$} & \multicolumn{5}{|c|}{$\overline{\text { Early Sy1 }}$} & \multicolumn{5}{|c|}{$\overline{\text { Late Sy1 }}$} \\
\hline & $N$ & $f(\%)$ & $\sigma$ & $\langle\Sigma\rangle$ & $\sigma$ & $N$ & $f(\%)$ & $\sigma$ & $\langle\Sigma\rangle$ & $\sigma$ & $N$ & $f(\%)$ & $\sigma$ & $\langle\Sigma\rangle$ & $\sigma$ \\
\hline all & 725 & 100.0 & - & 1.8 & $\overline{0.1}$ & 553 & 100.0 & - & 1.9 & 0.1 & 71 & 100.0 & - & 1.6 & $\overline{0.2}$ \\
\hline$N_{\text {neigh }}=0$ & 170 & 23.0 & 2.0 & - & - & 128 & 23.1 & 2.3 & - & - & 14 & 20.0 & 6.0 & - & - \\
\hline$N_{\text {neigh }}=1$ & 156 & 22.0 & 2.0 & 0.9 & 0.2 & 115 & 20.8 & 2.1 & 1.1 & 0.2 & 21 & 30.0 & 7.0 & 0.6 & 0.3 \\
\hline $2 \leq N_{\text {neigh }} \leq 10$ & 350 & 48.0 & 3.0 & 1.9 & 0.1 & 273 & 49.4 & 3.7 & 1.9 & 0.1 & 35 & 49.0 & 10.0 & 1.7 & 0.2 \\
\hline $11 \leq N_{\text {neigh }} \leq 20$ & 33 & 4.6 & 0.8 & 4.7 & 0.1 & 26 & 4.7 & 0.9 & 4.6 & 0.1 & 1 & 1.0 & 1.0 & - & - \\
\hline $21 \leq N_{\text {neigh }} \leq 30$ & 4 & 0.6 & 0.3 & 7.7 & 0.4 & 3 & 0.5 & 0.3 & 8.3 & 0.9 & 0 & 0.0 & - & - & - \\
\hline$N_{\text {neigh }}>30$ & 2 & 0.3 & 0.2 & 13.0 & 2.0 & 1 & 0.2 & 0.2 & - & - & 0 & 0.0 & - & - & - \\
\hline$r_{\max } \leq 100 \mathrm{kpc}$ & 10 & 1.4 & 0.4 & 63.0 & 24.0 & 7 & 1.3 & 0.5 & 60.0 & 21.0 & 0 & 0.0 & - & - & - \\
\hline \multirow[t]{2}{*}{ Neighbours } & \multicolumn{5}{|c|}{ Sy2 } & \multicolumn{5}{|c|}{ Early Sy2 } & \multicolumn{5}{|c|}{ Late Sy2 } \\
\hline & $N$ & $\mathrm{f}(\%)$ & $\sigma$ & $\langle\Sigma\rangle$ & $\sigma$ & $N$ & $\mathrm{f}(\%)$ & $\sigma$ & $\langle\Sigma\rangle$ & $\sigma$ & $N$ & $\mathrm{f}(\%)$ & $\sigma$ & $\langle\Sigma\rangle$ & $\sigma$ \\
\hline all & 1104 & 100.0 & - & 1.6 & 0.1 & 297 & 100.0 & - & 1.9 & 0.1 & 628 & 100.0 & & 1.5 & 0.1 \\
\hline$N_{\text {neigh }}=0$ & 306 & 28.0 & 2.0 & - & - & 88 & 30.0 & 4.0 & - & - & 171 & 27.0 & 2.0 & - & - \\
\hline$N_{\text {neigh }}=1$ & 237 & 22.0 & 2.0 & 0.8 & 0.1 & 50 & 17.0 & 3.0 & 0.8 & 0.2 & 149 & 24.0 & 2.0 & 0.8 & 0.2 \\
\hline $2 \leq N_{\text {neigh }} \leq 10$ & 505 & 46.0 & 3.0 & 1.8 & 0.1 & 142 & 48.0 & 5.0 & 2.0 & 0.1 & 279 & 44.0 & 3.0 & 1.6 & 0.1 \\
\hline $11 \leq N_{\text {neigh }} \leq 20$ & 28 & 2.5 & 0.5 & 5.2 & 0.2 & 8 & 3.0 & 1.0 & 5.2 & 0.3 & 14 & 2.2 & 0.6 & 5.3 & 0.3 \\
\hline $21 \leq N_{\text {neigh }} \leq 30$ & 5 & 0.5 & 0.2 & 8.2 & 0.3 & 3 & 1.0 & 0.6 & 8.2 & 0.0 & 2 & 0.3 & 0.2 & 9.7 & 0.2 \\
\hline$N_{\text {neigh }}>30$ & 0 & 0.0 & - & - & - & 0 & 0.0 & - & - & - & 0 & 0.0 & - & - & - \\
\hline$r_{\max } \leq 100 \mathrm{kpc}$ & 23 & 2.1 & 0.4 & 84.0 & 64.0 & 6 & 2.0 & 0.8 & 117.0 & 16.0 & 13 & 2.1 & 0.6 & 71.0 & 20.0 \\
\hline \multirow[t]{2}{*}{ Neighbours } & \multicolumn{5}{|c|}{ PGs (early) } & \multicolumn{5}{|c|}{ UELGs (late) } & \multicolumn{5}{|c|}{ SFGs (late) } \\
\hline & $N$ & $f(\%)$ & $\sigma$ & $\langle\Sigma\rangle$ & $\sigma$ & $N$ & $f(\%)$ & $\sigma$ & $\langle\Sigma\rangle$ & $\sigma$ & $N$ & $f(\%)$ & $\sigma$ & $\langle\Sigma\rangle$ & $\sigma$ \\
\hline$\overline{a l l}$ & 8144 & 100.0 & & 2.5 & $\overline{0.0}$ & 6955 & 100.0 & & 1.6 & $\overline{0.0}$ & 4837 & 100.0 & & 1.5 & $\overline{0.0}$ \\
\hline$N_{\text {neigh }}=0$ & 1573 & 19.3 & 0.5 & - & - & 2192 & 31.5 & 0.8 & - & - & 1749 & 36.0 & 1.0 & - & - \\
\hline$N_{\text {neigh }}=1$ & 1240 & 15.2 & 0.5 & 0.9 & 0.1 & 1449 & 20.8 & 0.6 & 0.8 & 0.0 & 1083 & 22.4 & 0.8 & 0.8 & 0.1 \\
\hline $2 \leq N_{\text {neigh }} \leq 10$ & 3996 & 49.1 & 0.9 & 2.2 & 0.0 & 2940 & 42.3 & 0.9 & 1.7 & 0.0 & 1777 & 37.0 & 1.0 & 1.6 & 0.0 \\
\hline $11 \leq N_{\text {neigh }} \leq 20$ & 913 & 11.2 & 0.4 & 5.1 & 0.0 & 276 & 4.0 & 0.2 & 4.7 & 0.1 & 104 & 2.2 & 0.2 & 5.0 & 0.1 \\
\hline $21 \leq N_{\text {neigh }} \leq 30$ & 263 & 3.2 & 0.2 & 8.3 & 0.1 & 38 & 0.5 & 0.1 & 7.8 & 0.1 & 18 & 0.4 & 0.1 & 8.1 & 0.2 \\
\hline$N_{\text {neigh }}>30$ & 71 & 0.9 & 0.1 & 11.8 & 0.2 & 3 & 0.0 & 0.0 & 11.3 & 0.3 & 2 & 0.0 & 0.0 & 11.2 & 0.0 \\
\hline$r_{\max } \leq 100 \mathrm{kpc}$ & 88 & 1.1 & 0.1 & 67.0 & 40.0 & 57 & 0.8 & 0.1 & 52.0 & 133.0 & 104 & 2.2 & 0.2 & 72.0 & 32.0 \\
\hline
\end{tabular}

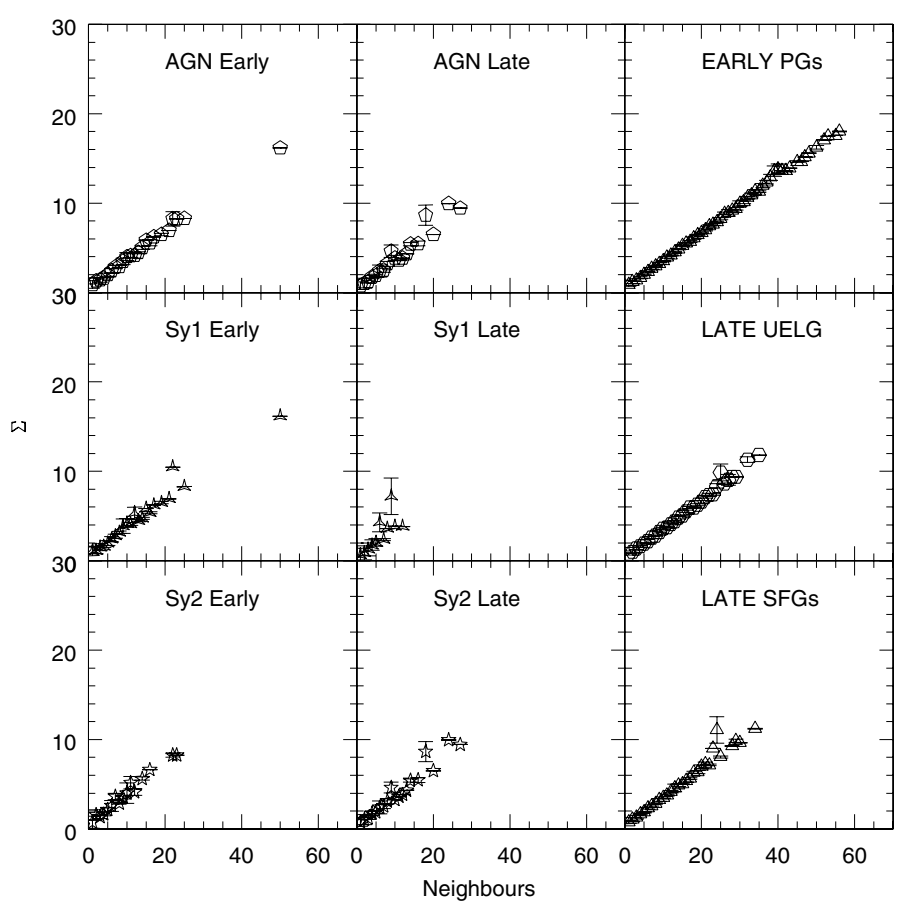

Fig. 10. Comparison of the environmental properties in AGNs and control samples.

The environments of AGNs, SFGs, and PGs have been compared by defining a median density parameter $\langle\Sigma\rangle$. The comparison of AGNs with normal galaxies was made through matching the morphological types and the distribution of the galaxy diameters. The main results are:

1. The fraction of galaxies classified as an AGNs is $2 \%$. This is probably a lower limit due to the severe selection criteria. The fraction of SFGs is 7\% and the fraction of PGs is $18 \%$. UELGs, that is, emission-line galaxies that could not be classified as SFGs or AGNs, are $\sim 55 \%$.

2. There is no evidence for a difference in the fraction of neighbour galaxies in Sy1 compared to Sy2. The ratio of Sy1 to Sy2 does not change if we take into account all systems, or isolated/pair systems only, in accordance with the unified model. The median surface density is the same for Sy 2 and Sy1 $(\langle\Sigma\rangle \sim 2)$. The comparison with control samples of PGs, UELGs, and SFGs does not indicate any significative difference in the environment. with the exception of close systems $\left(r_{\max } \leq 100 \mathrm{kpc}\right)$ : we find a higher fraction of Sy2 in close pairs $(\sim 2 \%)$, similar to SFGs, than Sy1 $(\sim 1 \%)$.

3 . The analysis of the frequency of systems with close neighbours in Sy1 and Sy2, before and after the morphological separation, shows that their distribution is different only for pairs. If we do not include pairs, the distribution is the same in Sy1 and Sy2 and is consistent with that in SFGs. This would imply a higher probability of finding Sy2 than Sy1 in close pairs.

We conclude that in our sample there is no evidence for a difference in the large-scale environment between Sy1 and Sy2 galaxies. The only difference is found in close pairs, even if the numbers are low (21 Sy2, 10 Sy1). If these systems are interacting galaxies, the lower fraction of Sy1 may be due to an increased 
Table 3. Environmental properties for AGNs and control samples of normal galaxies with the same distribution in the galaxy diameter. The number $(N)$ of galaxies with at least one companion within $100 \mathrm{kpc}$, the fraction $(f)$ of galaxies with the associated errors $(\sigma)$

\begin{tabular}{|c|c|c|c|c|c|c|c|c|c|}
\hline \multirow[t]{2}{*}{ Neighbours } & \multicolumn{3}{|c|}{$\overline{\text { Sy1 }}$} & \multicolumn{3}{|c|}{ Early Sy1 } & \multicolumn{3}{|c|}{$\overline{\text { Late Sy1 }}$} \\
\hline & $N$ & $f(\%)$ & $\sigma$ & $N$ & $f(\%)$ & $\sigma$ & $N$ & $f(\%)$ & $\sigma$ \\
\hline all & 82 & 11.0 & 1.0 & 69 & 13.0 & 2.0 & 5 & 7.0 & 3.0 \\
\hline$N_{\text {neigh }}=1$ & 10 & 1.4 & 0.4 & 7 & 1.3 & 0.5 & 0 & 0.0 & - \\
\hline$N_{\text {neigh }}=2$ & 6 & 0.8 & 0.3 & 4 & 0.7 & 0.3 & 0 & 0.0 & - \\
\hline $3 \leq N_{\text {neigh }} \leq 10$ & 53 & 7.0 & 1.0 & 47 & 9.0 & 1.0 & 4 & 6.0 & 3.0 \\
\hline $11 \leq N_{\text {neigh }} \leq 20$ & 10 & 1.4 & 0.4 & 9 & 1.6 & 0.5 & 1 & 1.0 & 1.0 \\
\hline $21 \leq N_{\text {neigh }} \leq 30$ & 3 & 0.4 & 0.2 & 2 & 0.4 & 0.2 & 0 & 0.0 & - \\
\hline$N_{\text {neigh }}>30$ & 0 & 0.0 & - & 0 & 0.0 & - & 0 & 0.0 & - \\
\hline \multirow[t]{2}{*}{ Neighbours } & \multicolumn{3}{|c|}{ Sy2 } & \multicolumn{3}{|c|}{ Early Sy2 } & \multicolumn{3}{|c|}{ Late Sy2 } \\
\hline & $N$ & $f(\%)$ & $\sigma$ & $N$ & $f(\%)$ & $\sigma$ & $N$ & $f(\%)$ & $\sigma$ \\
\hline all & 101 & 9.1 & 0.9 & 35 & 12.0 & 2.0 & 52 & 8.0 & 1.0 \\
\hline$N_{\text {neigh }}=1$ & 21 & 1.9 & 0.4 & 6 & 2.0 & 0.8 & 11 & 1.7 & 0.5 \\
\hline$N_{\text {neigh }}=2$ & 20 & 1.8 & 0.4 & 3 & 1.0 & 0.5 & 15 & 2.4 & 0.6 \\
\hline $3 \leq N_{\text {neigh }} \leq 10$ & 48 & 4.3 & 0.6 & 20 & 7.0 & 2.0 & 22 & 3.5 & 0.7 \\
\hline $11 \leq N_{\text {neigh }} \leq 20$ & 7 & 0.6 & 0.2 & 2 & 0.6 & 0.4 & 3 & 0.5 & 0.3 \\
\hline $21 \leq N_{\text {neigh }} \leq 30$ & 5 & 0.4 & 0.2 & 4 & 1.3 & 0.6 & 1 & 0.1 & 0.1 \\
\hline$N_{\text {neigh }}>30$ & 0 & 0.0 & - & 0 & 0.0 & - & 0 & 0.0 & - \\
\hline \multirow{2}{*}{ Neighbours } & \multicolumn{3}{|c|}{ PGs (early) } & \multicolumn{3}{|c|}{ UELGs (late) } & \multicolumn{3}{|c|}{ SFGs (late) } \\
\hline & $N$ & $f(\%)$ & $\sigma$ & $N$ & $f(\%)$ & $\sigma$ & $N$ & $f(\%)$ & $\sigma$ \\
\hline all & 1126 & 13.8 & 0.4 & 383 & 5.5 & 0.2 & 357 & 7.4 & 0.4 \\
\hline$N_{\text {neigh }}=1$ & 84 & 1.0 & 0.1 & 57 & 0.8 & 0.1 & 102 & 2.1 & 0.2 \\
\hline$N_{\text {neigh }}=2$ & 95 & 1.2 & 0.1 & 51 & 0.7 & 0.1 & 81 & 1.7 & 0.2 \\
\hline $3 \leq N_{\text {neigh }} \leq 10$ & 539 & 6.6 & 0.3 & 224 & 3.2 & 0.2 & 139 & 2.9 & 0.2 \\
\hline $11 \leq N_{\text {neigh }} \leq 20$ & 244 & 3.0 & 0.2 & 39 & 0.6 & 0.1 & 31 & 0.6 & 0.1 \\
\hline $21 \leq N_{\text {neigh }} \leq 30$ & 117 & 1.4 & 0.1 & 10 & 0.10 & 0.04 & 1 & 0.02 & 0.02 \\
\hline$N_{\text {neigh }}>30$ & 47 & 0.5 & 0.1 & 2 & 0.03 & 0.02 & 3 & 0.06 & 0.03 \\
\hline
\end{tabular}

probability of molecular gas being driven towards the nucleus obscuring the broad line region, as proposed by Dultzin-Hacyan et al. (2003). This result does not seem compatible with the simplest formulation of the unified model for Seyfert galaxies, where both type 1 and 2 should be intrinsically alike, the only difference being the result of the orientation of an obscuring torus with respect to the line of sight. A more detailed analysis of these systems will be the subject of a future paper.

Acknowledgements. We thank the anonymous referee for the useful comments that improved the paper.

We are grateful to Chris P. Haines for having carefully read the manuscript.

G.S. acknowledges the financial support from Regione Campania through the Research Contract for the project Evolution of Normal and Active Galaxies.

The SDSS is managed by the Astrophysical Research Consortium (ARC) for the Participating Institutions. The Participating Institutions are: the University of Chicago, Fermilab, the Institute for Advanced Study, the Japan Participation Group, John Hopkins University, Los Alamos National Laboratory, the Max Planck Institute for Astronomy (MPIA), the Max Planck Institute for Astrophysics (MPA), New Mexico State University, University of Pittsburgh, Princeton University, the United States Naval Observatory, and the University of Washington. Funding for the creation and distribution of the SDSS Archive has been provided by the Alfred P. Sloan Foundation, the Participating Institutions, the National Aeronautics and Space Administration, the National Science Foundation, the US Department of Energy, the Japanese Monbukagakusho, and the Max Planck Society. The SDSS web site is: http://www.sdss.org.

This work is partially supported by the EC contract HPRN-CT-2002-00316 (SISCO network), by MIUR-COFIN-2003 n. 2003020150_004, and by MIUR-COFIN-2004 No. 2004020323_001.

\section{References}

Abazajian, K., Adelman-McCarthy, J. K., Agüeros, M. A., et al. 2004, AJ, 128, 502 Abell, G. O. 1958, ApJ, 3, 211

Antonucci, R. 1993, ARA\&A, 31, 473

Balogh, M., Eke, V., Miller, C., et al. 2004, MNRAS, 348, 1355

Benson, A. J., Frenk, C. S., \& Sharples, R. M. 2002, ApJ, 574, 104

Bernardi, M., Steth, R. K., Nichol, R. C., et al. 2005, AJ, 129, 61

Blanton, M. R., Dalcanton, J., Eisenstein, D., et al. 2001, AJ, 121, 2358

Blanton, M. R., Brinkmann, J., Csabai, I., et al. 2003a, AJ, 125, 2348
Blanton, M. R., Lin, H., Lupton, R. H., et al. 2003b, AJ, 125, 2276 Brown, M. J. I., Boyle, B. J., \& Webster, R. L. 2001, AJ, 121, 2381 Carter, B. J., Fabricant, D. G., Geller, M. J., et al. 2001, ApJ, 559, 606 Colless, M., Dalton, G., Maddox, S., et al. 2001, MNRAS, 328, 1039 Dahari, O. 1984, AJ, 89, 966

Dahari, O. 1985, AJ, 90, 1772

de Robertis, M. M., Yee, H. K. C., \& Hayhoe, K. 1998, ApJ, 496, 93

Dressler, A. 1980, ApJ, 236, 351

Dressler, A., Thompson, I. B., \& Shectman, S. A. 1985, ApJ, 288, 481

Dultzin-Hacyan, D., Krongold, Y., \& Marziani, P. 2003, Rev. Mex. Astron. Astrofis., 17, 79 Dultzin-Hacyan, D., Krongold, Y., Fuentes-Guridi, I., \& Marziani, P. 1999, ApJ, 513, 111 Fadda, D., Girardi, M., Giuricin, G., Mardirossian, F., \& Mezzetti, M. 1996, ApJ, 473, 670 Fuentes-Williams, T. \& Stocke, J. T. 1988, AJ, 96, 1235

Fukugita, M., Ichikawa, T., Gunn, J. E., et al. 1996, AJ, 111, 1748

Gómez, P. L., Nichol, R. C., Miller, C. J., et al. 2003, ApJ, 584, 210

Gunn, J. E., Carr, M., Rockosi, C., et al. 1998, AJ, 116, 3040

Huchra, J. P., \& Burg, R. 1992, ApJ, 393, 90

Ivezić, Z., Menou, K., Knapp, G. R., et al. 2002, AJ, 124, 2364

Kauffmann, G., Coldberg, J. M., Diaferio, A., \& White, S. D. M. 1999, MNRAS, 303, 188 Kewley, L. J., Dopita, M. A., Sutherland, R. S., et al. 2001, ApJ, 556, 121

Krongold, Y., Dultzin-Hacyan, D., \& Marziani, P. 2002, AJ, 572, 169

Koulouridis, E., Plionis, M.,Chavushyan, V., et al. 2005 [arXiv: astro-ph/0509843]

Lambas, D. G., Tissera, P. B., Alonso, M. S., \& Coldwell, G. 2003, MNRAS, 346, 1189

Laurikainen, E., \& Salo, H. 1995, A\&AS, 293, 683

Laurikainen, E., Salo, H., Teerikorpi, P., \& Petrov, G. 1994, A\&AS, 108, 491

Maia, M. A. G., Willmer, C. N. A., Rossetto, B. M., \& Machado, R. S. 2004, Proceed. IAU Symp., 124

Martini, P., Regan, M. W., Mulchaey, J. S., \& Pogge, R. W. 2003, ApJ, 589, 774

Miller, C. J., Nichol, R. C., Gómez, P. L., Hopkins, A. M., \& Bernardi, M. 2003, ApJ, 597, 142

Nikolic, B., Cullen, H., \& Alexander, P. 2004, MNRAS, 355, 874

Rafanelli, P., Violato, M., \& Baruffolo, A. 1995, AJ, 109, 1546

Schmitt, H. R. 2001, AJ, 122, 2243

Schreier, E. J., Koekemoer, A. M., Grogin, N. A., et al. 2001, ApJ, 560, 127

Shectman, S. A., Landy, S. D., Oemler, A., et al. 1996, ApJ, 470, 172

Sorrentino, G., Kelm, B., \& Focardi, P. 2003, otmu.conf, 333

Stauffer, J. R. 1982, ApJ, 262, 66

Storchi-Bergmann, T., Delgado, R. M. G., Schmitt, H. R., \& Heckman, T. 2001, ApJ, 559, 147

Strauss, M. A., Weinberg, D. H., Lupton, R. H., et al. 2002, AJ, 124, 1810

Tran, H.D. 2003, ApJ, 583, 632

Walker, I. R., Mihos, J. C., \& Hernquist, L. 1996, ApJ, 460, 121

Wilman, D. J., Balogh, M. L., Bower, R. G., et al. 2005, MNRAS, 358, 71

York, D. G., Adelman, J., Anderson, John, E. jr, et al. 2000, AJ, 120, 1579 